\title{
On Lie Algebroids and Poisson Algebras
}

\author{
Dennise GARCÍA-BELTRÁN ${ }^{\dagger}$, José A. VALLEJO ${ }^{\dagger}$ and Yuriu VOROBJEV $V^{\ddagger}$ \\ ${ }^{\dagger}$ Facultad de Ciencias, Universidad Autónoma de San Luis Potosí, México \\ E-mail: dennise.gb@alumnos.uaslp.edu.mx, jvallejo@fc.uaslp.mx \\ URL: http://galia.fc.uaslp.mx/ jvallejo/ \\ $\ddagger$ Departamento de Matemáticas, Universidad de Sonora, México \\ E-mail: yurimv@guaymas.uson.mx
}

Received June 08, 2011, in final form February 01, 2012; Published online February 10, 2012

http://dx.doi.org/10.3842/SIGMA.2012.006

\begin{abstract}
We introduce and study a class of Lie algebroids associated to faithful modules which is motivated by the notion of cotangent Lie algebroids of Poisson manifolds. We also give a classification of transitive Lie algebroids and describe Poisson algebras by using the notions of algebroid and Lie connections.
\end{abstract}

Key words: transitive Lie algebroids; Lie-Rinehart algebras; Poisson brackets; algebraic connections

2010 Mathematics Subject Classification: 53D17; 17B63

\section{Introduction}

Let $(M,\{\cdot, \cdot\})$ be a Poisson manifold equipped with the bracket $\{\cdot, \cdot\}$, which is determined by a Poisson bivector $\mathbf{P} \in \Gamma \Lambda^{2} T M$. It is well known that the cotangent bundle $T^{*} M$ carries a natural Lie algebroid structure (see Section 2 for definitions), that is, on differential 1-forms (sections of $\left.T^{*} M\right)$ the following bracket can be defined (see [1, Proposition 14.19]),

$$
\llbracket \alpha, \beta \rrbracket=i_{P \alpha} \mathrm{d} \beta-i_{P \beta} \mathrm{d} \alpha+\mathrm{d}(\beta(P \alpha)),
$$

where $P: T^{*} M \rightarrow T M$ is the vector bundle morphism canonically induced by $\mathbf{P}$ through $\beta(P \alpha)=\mathbf{P}(\alpha, \beta)$. Moreover, the following Leibniz-like rule is satisfied, for any smooth function $f \in \mathcal{C}^{\infty}(M)$ :

$$
\llbracket \alpha, f \beta \rrbracket=f \llbracket \alpha, \beta \rrbracket+(P \alpha)(f) \cdot \beta .
$$

That means that the corresponding anchor map is just $P$. Note that in the case when $\alpha, \beta$ in (1) are exact $\left(\alpha=\mathrm{d} f\right.$ and $\beta=\mathrm{d} g$ for some $f, g \in \mathcal{C}^{\infty}(M)$ ), we have $\mathbf{P}(\alpha, \beta)=\{f, g\}$ and formula (1) reads:

$$
\llbracket \mathrm{d} f, \mathrm{~d} g \rrbracket=\mathrm{d}\{f, g\} .
$$

Moreover, the bracket (1) of two closed forms is again closed.

In fact, the properties above characterize the Lie algebroid structure on the cotangent bundle that comes from a Poisson bracket on the base manifold (see [11, 7]). More precisely, given a Lie algebroid $\left(T^{*} M, \llbracket \cdot, \cdot \rrbracket, \rho\right)$ on the cotangent bundle $T^{*} M$, there exists a Poisson bracket $\{\cdot, \cdot\}$ on $M$ such that $\rho=P$ if and only if the following conditions are satisfied:

(a) $\rho$ is skew-symmetric, i.e. $\beta(\rho(\alpha))=-\alpha(\rho(\beta))$, for all $\alpha, \beta \in \Omega^{1}(M)$.

(b) If $\alpha, \beta \in \Omega^{1}(M)$ are closed, then $\llbracket \alpha, \beta \rrbracket$ is also closed. 
The aim of the present work is to put the study of cotangent Lie algebroids of Poisson manifolds in an algebraic framework. In the first part (Section 3), we study and characterize a class of Lie algebroids with properties similar to (a), (b), which we call Lie algebroids of Poisson type. To this end, we will work in a slightly more general context than that of vector bundles over manifolds, using the notion of Lie-Rinehart algebra (see [11, 12]), also called a Lie pseudoalgebra (see [17, 20, 21, 22] and, particularly, [16] for many interesting remarks on the evolution of these notions and as a general reference), although we will continue using the denomination "Lie algebroid" for them (as they are the algebraic version of the geometric Lie algebroids). The dictionary in Example 1 can be used at any time to obtain the corresponding expressions for geometric Lie algebroids. We give several examples illustrating the different situations that can appear.

In the second part (Sections 4 and 5), we deepen in the relationship between transitive algebroids and Poisson structures for a certain class of spaces, those of the form $\operatorname{Der}(\mathcal{A}) \oplus V$, where $\mathcal{A}$ is a commutative algebra and $V$ an $\mathcal{A}$-module. We describe parametrizations of the transitive Lie algebroids on $\operatorname{Der}(\mathcal{A}) \oplus V$ following the techniques exposed in [24, 23], which are based on the use of a connection on a Lie algebroid. For completeness, we include a subsection in the preliminaries devoted to the topic of connections in an algebraic setting. Once the parametrization is given, we apply it to prove that a transitive algebroid endowed with a connection is isomorphic to one of the form $\operatorname{Der}(\mathcal{A}) \oplus V$. Finally, we obtain new classes of Poisson algebras on $\mathcal{A} \oplus V$ starting from Poisson algebras on $\mathcal{A}$.

\section{Preliminaries}

Throughout the document, unless otherwise explicitly stated, $\mathcal{A}$ denotes an associative, commutative algebra with identity element $1_{\mathcal{A}}$, over a commutative ring $\mathcal{R}$ with identity element $1_{\mathcal{R}}$.

\subsection{Derivations and connections in commutative algebras}

In subsequent sections, we will need to introduce connections on an algebroid. In our algebraic setting, the most appropriate notion of connection is Koszul's one, which is given in terms of derivations.

Definition 1. A derivation of the algebra $\mathcal{A}$ over $\mathcal{R}$ is a map $X \in \operatorname{Hom}_{\mathcal{R}}(\mathcal{A}, \mathcal{A})$ satisfying the Leibniz rule

$$
X(f \cdot g)=X(f) \cdot g+f \cdot X(g) .
$$

The set of derivations of $\mathcal{A}$ over $\mathcal{R}$ is denoted $\operatorname{Der}_{\mathcal{R}}(\mathcal{A})$ or simply $\operatorname{Der}(\mathcal{A})$ when there is no risk of confusion about the ring $\mathcal{R}$.

Remark 1. The definition just given can be extended to the case of derivations of $\mathcal{A}$ over $\mathcal{R}$ with values in an $\mathcal{A}$-module $M$. These are Abelian groups morphisms $X: \mathcal{A} \rightarrow M$ satisfying the Leibniz rule above, and form an $\mathcal{A}$-module denoted $\operatorname{Der}_{\mathcal{R}}(\mathcal{A}, M)$ or $\operatorname{simply} \operatorname{Der}(\mathcal{A}, M)$.

The set $\operatorname{Der}(\mathcal{A})$ has an $\mathcal{R}$-Lie algebra structure when endowed with the commutator of endomorphisms, given by $[X, Y]=X \circ Y-Y \circ X$.

Note also that, if $\mathcal{A}$ as an $\mathcal{R}$-module is faithful, then for every $X \in \operatorname{Der}(\mathcal{A})$ we have $X\left(1_{\mathcal{A}}\right)=0$ and indeed $X(r)=0$ for every $r \in \mathcal{R}$ viewed as a subalgebra of $\mathcal{A}$.

Definition 2. Let $M$ be an unitary $\mathcal{A}$-module. A derivation law, or Koszul connection, on $M$ is an $\mathcal{A}$-linear mapping $\nabla: \operatorname{Der}(\mathcal{A}) \rightarrow \operatorname{Hom}_{\mathcal{R}}(M, M)$ (the image of $X \in \operatorname{Der}(\mathcal{A})$ denoted $\left.\nabla_{X}\right)$ such that

$$
\nabla_{X}(f \cdot m)=X(f) \cdot m+f \cdot \nabla_{X}(m) .
$$


Not every $\mathcal{A}$-module $M$ admits a connection in this sense, but it is easy to see that any free $\mathcal{A}$-module does. Of course, arbitrary $\mathcal{A}$-modules do not need to be free. So, in order to obtain a big enough class of modules for which we can guarantee the existence of a Koszul connection, we will make a brief digression on modules of differentials and Connes connections (see [4]).

Let $\Omega^{1}(\mathcal{A})$ be the $\mathcal{A}$-module defined by the kernel of the multiplication $\mathcal{A} \otimes_{\mathcal{R}} \mathcal{A} \rightarrow \mathcal{A}$. Define the map d : $\mathcal{A} \rightarrow \Omega^{1}(\mathcal{A})$ by $\mathrm{d} a=1 \otimes a-a \otimes 1$, which is a derivation of $\mathcal{A}$ over $\mathcal{R}$ with values into $\Omega^{1}(\mathcal{A})$. It is clear from the definition that $\Omega^{1}(\mathcal{A})=\operatorname{Span}_{\mathcal{A}}\{\mathrm{d} f: f \in \mathcal{A}\}$ : Since the elements of $\Omega^{1}(\mathcal{A})$ lie in the kernel of the multiplication map, if $\sum a_{j} \otimes b_{j} \in \Omega^{1}(\mathcal{A})$, then $\sum a_{j} b_{j}=0$ and therefore

$$
\sum a_{j} \otimes b_{j}=\sum\left(a_{j} \otimes b_{j}-a_{j} b_{j} \otimes 1\right)=\sum a_{j} \mathrm{~d} b_{j}
$$

In fact, $\Omega^{1}(\mathcal{A})$ is the submodule of $C^{1}(\operatorname{Der}(\mathcal{A}), \mathcal{A})$ (the 1-component of the differential algebra $C(\operatorname{Der}(\mathcal{A}), \mathcal{A})$ of Chevalley-Eilenberg cochains of the Lie algebra $\operatorname{Der}(\mathcal{A})$ with values in the $\operatorname{Der}(\mathcal{A})$-module $\mathcal{A}$ ) generated by the elements $\mathrm{d} f, f \in \mathcal{A}$ (see [6]). Note, in particular, that this implies $\Omega^{1}(\mathcal{A}) \subset \operatorname{Der}^{*}(\mathcal{A})$.

Definition 3. Let $M$ be an $\mathcal{A}$-module. A Connes connection on $M$ is an $\mathcal{A}$-linear map $\delta: M \rightarrow$ $\Omega^{1}(\mathcal{A}) \otimes_{\mathcal{A}} M$ such that, for all $f \in \mathcal{A}, m \in M$,

$$
\delta(f m)=f \delta(m)+\mathrm{d} f \otimes_{\mathcal{A}} m .
$$

Remark 2. Connes' definition of a connection (see [4]) actually does not require that $\mathcal{A}$ be a commutative algebra. The definition goes back to a work by N. Katz [14].

Starting from a Connes connection, we can obtain a Koszul one. If $X \in \operatorname{Der}(\mathcal{A})$, then we define a right $\mathcal{A}$-linear pairing $\varphi: \operatorname{Der}(\mathcal{A}) \otimes_{\mathcal{R}} \Omega^{1}(\mathcal{A}) \rightarrow \mathcal{A}$ by

$$
\left(X, \sum a_{j} \mathrm{~d} b_{j}\right) \mapsto \sum a_{j} X\left(b_{j}\right)
$$

The Koszul connection $\nabla$ associated to $\delta$ can be constructed as follows: for $X \in \operatorname{Der}(\mathcal{A})$, $\nabla_{X} \in \operatorname{Hom}_{\mathcal{R}}(M, M)$ is the map given by applying the connection $\delta$ and then contracting the $\Omega^{1}(\mathcal{A})$ component with $\varphi$. Thus, if $m \in M$ is such that $\delta(m)=\sum a_{j} \mathrm{~d} b_{j} \otimes m_{j}$, for certain $a_{j}, b_{j} \in \mathcal{A}$ and $m_{j} \in M$, we have for each $f \in \mathcal{A}$ that $\delta(f m)=f \sum a_{j} \mathrm{~d} b_{j} \otimes m_{j}+\mathrm{d} f \otimes m$, and

$$
\begin{aligned}
\nabla_{X}(f m) & =\sum \varphi\left(X, f a_{j} \mathrm{~d} b_{j}\right) m_{j}+\varphi(X, d f) m=f \sum a_{j} X\left(b_{j}\right) m_{j}+X(f) m \\
& =f \nabla_{X}(m)+X(f) m .
\end{aligned}
$$

A basic result obtained by J. Cuntz and D. Quillen (see [5]) is that Connes connections on an $\mathcal{A}$-module $M$ are in bijective correspondence with $\mathcal{A}$-linear splittings of the natural action $\mathcal{A} \otimes_{\mathcal{R}} M \rightarrow M$. As a consequence, $M$ admits a Connes connection if and only if it is projective.

As said earlier, we will need later on to work with (Koszul) connections, so we need conditions on $\mathcal{A}$ to assure their existence. From what we have seen, these connections exist on any $\mathcal{A}$ module $M$ which is free or projective. Indeed, note that a free module is always projective, but there are projective modules which are not free. In the literature, there are several well-known conditions on $\mathcal{A}$ guaranteeing the projective character of $M$ (for example, that $\mathcal{A}$ be semi-simple as a ring). When we talk of a connection on $M$, unless otherwise explicitly stated, we will mean that any one of these conditions is satisfied and that the connection is Koszul. 


\subsection{Lie algebroids}

Definition 4. Let $\mathcal{F}$ be a faithful $\mathcal{A}$-module. A Lie algebroid (on $\mathcal{F}$ over the commutative algebra $\mathcal{A})$ is a triple $(\mathcal{F}, \llbracket \cdot, \cdot \rrbracket, \rho)$, where $\llbracket \cdot, \cdot \rrbracket$ is a Lie bracket on $\mathcal{F}$ and $\rho: \mathcal{F} \rightarrow \operatorname{Der}(\mathcal{A})$ is a morphism of $\mathcal{A}$-modules, called the anchor map, such that

$$
\llbracket X, f Y \rrbracket=f \llbracket X, Y \rrbracket+\rho(X)(f) Y,
$$

for all $f \in \mathcal{A}$ and for all $X, Y \in \mathcal{F}$.

Remark 3. Sometimes, the condition that the anchor map be a morphism of Lie algebras is included in the definition of Lie algebroid. However, this fact is a consequence of the conditions in Definition 4, as it has been noted by J.C. Herz, Y. Kosmann-Schwarzbach, F. Magri and J. Grabowski among others (see [9, 10, 17, 8]).

Definition 5. Let $(\mathcal{F}, \llbracket \cdot, \cdot \rrbracket, \rho)$ and $\left(\mathcal{F}^{\prime}, \llbracket \cdot, \cdot \rrbracket^{\prime}, \rho^{\prime}\right)$ be Lie algebroids (over the same algebra $\mathcal{A}$ and the same ring $\mathcal{R}$ ). A morphism of Lie algebroids is a morphism of $\mathcal{A}$-modules $\phi: \mathcal{F} \rightarrow \mathcal{F}^{\prime}$ such that

$$
\rho^{\prime} \circ \phi=\rho \quad \text { and } \quad \phi(\llbracket X, Y \rrbracket)=\llbracket \phi(X), \phi(Y) \rrbracket^{\prime},
$$

for all $X, Y \in \mathcal{F}$.

Let us consider some examples. The first is the classical one.

Example 1. Let $M$ be a manifold. Let $E \stackrel{\pi}{\rightarrow} M$ be a vector bundle over $M$ and $\mathcal{F}=\Gamma(E)$ the $\mathcal{C}^{\infty}(M)$-module of sections of $E$ (i.e. $\mathcal{A}=\mathcal{C}^{\infty}(M)$ and $\mathcal{R}=\mathbb{R}$ ). Then, the Lie algebroid structure on $\Gamma(E)$ is defined by a Lie bracket $\llbracket \cdot, \cdot \rrbracket$ on $\Gamma(E)$ with an anchor map

$$
q: \Gamma(E) \rightarrow \operatorname{Der}_{\mathbb{R}} \mathcal{C}^{\infty}(M) \cong \Gamma(T M),
$$

such that for all $f \in \mathcal{C}^{\infty}(M)$ and for all $X, Y \in \Gamma(E)$ :

1) $\llbracket X, f Y \rrbracket=f \llbracket X, Y \rrbracket+q(X)(f) Y$,

2) $q(f X+Y)=f q(X)+q(Y)$.

In particular, if $E=T^{*} M$, then the Lie algebroid structure is given by the bracket (1) where the anchor is the Poisson mapping $P$. For $E=T M$ we have the trivial Lie algebroid, where $q=\mathrm{Id}_{T M}$.

Example 2. Consider the $\mathcal{R}$-algebra of dual numbers over $\mathcal{A}$,

$$
\mathcal{A}^{\prime}=\mathcal{A}[\theta]=\left\{x+y \theta: x, y \in \mathcal{A}, \theta^{2}=0\right\},
$$

with the obvious operations. Clearly, $\mathcal{A}^{\prime}$ is an $\mathcal{A}^{\prime}$-module and we can endow it with the Lie algebra structure given by the bracket:

$$
\llbracket x_{1}+y_{1} \theta, x_{2}+y_{2} \theta \rrbracket=\left(x_{1} y_{2}-y_{1} x_{2}\right) \theta
$$

for $x_{1}+y_{1} \theta, x_{2}+y_{2} \theta \in \mathcal{A}^{\prime}$. Thus $\left(\mathcal{A}^{\prime}, \llbracket \cdot, \cdot \rrbracket, \rho\right)$ is a Lie algebroid with anchor map

$$
\begin{aligned}
\rho: & \mathcal{A}^{\prime} \rightarrow \operatorname{Der}\left(\mathcal{A}^{\prime}\right), \\
x+y \theta & \longmapsto \operatorname{ad}_{x}
\end{aligned}
$$

for $x+y \theta \in \mathcal{A}^{\prime}$. Here $\operatorname{ad}_{x}\left(x_{1}+y_{1} \theta\right)=\llbracket x, x_{1}+y_{1} \theta \rrbracket$ is the adjoint map of $x=\operatorname{pr}_{1}(x+y \theta)$.

The next example will be relevant in Section 4.

Example 3. Consider the $\mathcal{A}$-module $\operatorname{Der}(\mathcal{A}) \oplus \mathcal{A}$. Denote by $\operatorname{pr}_{1}$ the projection onto the first factor, $\operatorname{pr}_{1}: \operatorname{Der}(\mathcal{A}) \oplus \mathcal{A} \rightarrow \operatorname{Der}(\mathcal{A})$, and define the following bracket:

$$
\llbracket\left(D_{1}, a_{1}\right),\left(D_{2}, a_{2}\right) \rrbracket=\left(\left[D_{1}, D_{2}\right], D_{1}\left(a_{2}\right)-D_{2}\left(a_{1}\right)\right)
$$

for $\left(D_{1}, a_{1}\right),\left(D_{2}, a_{2}\right) \in \operatorname{Der}(\mathcal{A}) \oplus \mathcal{A}$, where $\left[D_{1}, D_{2}\right]$ is the commutator of endomorphisms. Then, $\left(\operatorname{Der}(\mathcal{A}) \oplus \mathcal{A}, \llbracket \cdot, \cdot \rrbracket, \operatorname{pr}_{1}\right)$ is a Lie algebroid. 


\section{Lie algebroids of Poisson type}

Definition 6. A Poisson algebra $(\mathcal{A},\{\}$,$) is an associative algebra \mathcal{A}$ together with a Lie bracket which is also a derivation for the product in $\mathcal{A}$, that is, there is an $\mathcal{R}$-bilinear operation $\{\}:, \mathcal{A} \times \mathcal{A} \longrightarrow \mathcal{A}$ such that

1) $\{f, g\}=-\{g, f\}$ (skew-symmetry),

2) $\{f,\{g, h\}\}+\{g,\{h, f\}\}+\{h,\{f, g\}\}=0$ (Jacobi identity),

3) $\{f, g h\}=\{f, g\} h+g\{f, h\}$ (Leibniz rule),

for all $f, g, h \in \mathcal{A}$.

If $(\mathcal{A},\{\}$,$) is a Poisson algebra, then we can define the adjoint map ad : \mathcal{A} \longrightarrow \operatorname{Der}(\mathcal{A})$ by

$$
\operatorname{ad}(f)=\left\{f,_{-}\right\},
$$

for $f \in \mathcal{A}$. Then, extending the mapping $\mathrm{d} f \longmapsto \mathrm{ad}_{f}$ by linearity, we get a morphism $\rho$ : $\Omega^{1}(\mathcal{A}) \longrightarrow \operatorname{Der}(\mathcal{A})$ uniquely defined by

$$
\rho(\mathrm{d} f)=\operatorname{ad}_{f}, \quad \forall f \in \mathcal{A} .
$$

Sometimes (by analogy with Poisson manifolds), $a_{f}$ is referred to as the Hamiltonian vector field corresponding to $f \in \mathcal{A}$, and denoted by $X_{f}$ (we will use this notation and terminology later in Section 5).

Also, given an $\alpha \in \Omega^{1}(\mathcal{A})$, we can define $\mathrm{d} \alpha$ through the usual formula

$$
\mathrm{d} \alpha(X, Y)=X(\alpha(Y))-Y(\alpha(X))-\alpha([X, Y]),
$$

for all $X, Y \in \operatorname{Der}(\mathcal{A})$.

Theorem 1. If $(\mathcal{A},\{\}$,$) is a Poisson algebra, then \left(\Omega^{1}(\mathcal{A}),[],, \rho\right)$ is a Lie algebroid with anchor map $\rho$ defined by (2) and the Lie bracket

$$
\llbracket \alpha, \beta \rrbracket=\iota_{\rho(\alpha)} \mathrm{d} \beta-\iota_{\rho(\beta)} \mathrm{d} \alpha+\mathrm{d}(\beta(\rho(\alpha))) .
$$

Proof. All the required properties can be checked directly from the definition of the bracket (3). To verify the Jacobi identity, it is easier to consider a system $\left\{\mathrm{d} f_{i}\right\}_{i \in I}$ of generators of $\Omega^{1}(\mathcal{A})$, and to write the elements $\alpha \in \Omega^{1}(\mathcal{A})$ as $\alpha=g_{i} \mathrm{~d} f_{i}$ for some $g_{i} \in \mathcal{A}$.

Example 4. Let $(M,\{\}$,$) be a Poisson manifold, i.e., \left(\mathcal{C}^{\infty}(M),\{\},\right)$ is a Poisson algebra. Using Theorem 1 we have that $\Omega^{1}\left(\mathcal{C}^{\infty}(M)\right)=\Omega^{1}(M)$ is a Lie algebroid with anchor map $\rho=P$ (defined by (2)) and the bracket (1):

$$
\llbracket \alpha, \beta \rrbracket=\iota_{\rho(\alpha)} \mathrm{d} \beta-\iota_{\rho(\beta)} \mathrm{d} \alpha+\mathrm{d}(\beta(\rho(\alpha))),
$$

for all $\alpha, \beta \in \Omega^{1}(M)$. The proof is based on the fact that there exists a finite subset $\left\{g_{1}, \ldots, g_{k}\right\} \subset$ $C^{\infty}(M)$ (with $k \leq 2 \operatorname{dim} M+1$ ) such that the $C^{\infty}(M)$-module $\Omega^{1}(M)$ is spanned by $\left\{\mathrm{d} g_{1}, \ldots, \mathrm{d} g_{k}\right\}$. This, in turn, is a consequence of Whitney's embedding theorem and the fact that the sheaf of germs of smooth functions is soft (see [2]).

Theorem 1 tells us that given a Poisson algebra we have a Lie algebroid canonically associated to it. We are interested now in the reciprocal: When does a Lie algebroid $\left(\Omega^{1}(\mathcal{A}), \llbracket \cdot, \cdot \rrbracket, \rho\right)$ determine a Poisson structure on $\mathcal{A}$ ? 
Given a Lie algebroid $\left(\Omega^{1}(\mathcal{A}), \llbracket \cdot, \cdot \rrbracket, \rho\right)$, for any $f, g \in \mathcal{A}$ we can define

$$
\{f, g\}=(\mathrm{d} g)(\rho(\mathrm{d} f))=(\rho(\mathrm{d} f))(g) .
$$

The bracket $\{$,$\} defined in this way is clearly \mathcal{R}$-bilinear and satisfies the Leibniz rule

$$
\{f, g h\}=(\rho(d f))(g h)=g \rho(d f)(h)+\rho(d f)(g) h=g\{f, h\}+\{f, g\} h,
$$

for all $f, g, h \in \mathcal{A}$.

Thus, in order to get a Poisson structure on $\mathcal{A}$ we only need to take care of the skew-symmetry and the Jacobi identity.

Definition 7. The anchor map $\rho$ of a Lie algebroid $\left(\Omega^{1}(\mathcal{A}), \llbracket \cdot, \cdot \rrbracket, \rho\right)$ is said to be skew-symmetric if

$$
\alpha(\rho(\beta))=-\beta(\rho(\alpha))
$$

for all $\alpha, \beta \in \Omega^{1}(\mathcal{A})$.

If we assume that the anchor map is skew-symmetric, then the new operation defined by (4) is also skew-symmetric. Now, let us turn our attention to the Jacobi identity.

Theorem 2. Let $\left(\Omega^{1}(\mathcal{A}), \llbracket \cdot, \cdot \rrbracket, \rho\right)$ be a Lie algebroid, and define $Q \in \Lambda^{2}(\operatorname{Der}(\mathcal{A}))$ by $Q(\mathrm{~d} f, \mathrm{~d} g)=$ $\mathrm{d} g(\rho(\mathrm{d} f))$ for $f, g \in \mathcal{A}$. The following conditions are equivalent:

(i) the bracket $\{$,$\} defined by \{f, g\}=\rho(\mathrm{d} f)(g)$ satisfies the Jacobi identity,

(ii) $[Q, Q]_{\mathrm{SN}}=0$,

(iii) $[\rho(\mathrm{d} f), \rho(\mathrm{d} g)]=\rho(\mathrm{d}\{f, g\})$.

Here, $[\cdot, \cdot]_{\mathrm{SN}}$ denotes the Schouten-Nijenhuis bracket on multiderivations [15].

Proof. It is based on the independent computation of both sides of the equation $\frac{1}{2}\left[\mathcal{L}_{Q}, \iota_{Q}\right]=$ $\frac{1}{2} \iota_{[Q, Q]_{\mathrm{SN}}}$, where on the left-hand side we have the graded commutator of operators on the graded algebra $\Lambda(\mathcal{A})$. Note that due to the $\mathcal{A}$-linearity, we only need to apply these operators to basis elements.

This result motivates the following definition.

Definition 8. A Lie algebroid $\left(\Omega^{1}(\mathcal{A}), \llbracket \cdot, \cdot \rrbracket, \rho\right)$ is of Poisson type if:

1) the anchor $\rho$ is skew-symmetric,

2) one of the equivalent conditions (i)-(iii) in Theorem 2 holds.

In other words, a Lie algebroid $\left(\Omega^{1}(\mathcal{A}), \llbracket \cdot, \cdot \rrbracket, \rho\right)$ is of Poisson type if it determines a Poisson structure on the algebra $\mathcal{A}$.

Another important issue for us is to determine the form of the bracket of a Lie algebroid of Poisson type. We know that the classical example of Poisson manifolds leads to brackets of the type (1). The following result characterizes the class of such algebroids.

Proposition 1. If $\left(\Omega^{1}(\mathcal{A}), \llbracket \cdot, \cdot \rrbracket, \rho\right)$ is a Lie algebroid for which $\rho$ is skew-symmetric and $\llbracket \mathrm{d} f, \mathrm{~d} g \rrbracket=\mathrm{d}\{f, g\}$, then the bracket of the Lie algebroid is of the form

$$
\llbracket \alpha, \beta \rrbracket=\iota_{\rho(\alpha)} \mathrm{d} \beta-\iota_{\rho(\beta)} \mathrm{d} \alpha+\mathrm{d}(\beta(\rho(\alpha))) .
$$


Proof. Note first that, if $\alpha=\mathrm{d} f$ and $\beta=\mathrm{d} g$ for some $f, g, \in \mathcal{A}$, then

$$
\iota_{\rho(\alpha)} d \beta-\iota_{\rho(\beta)} \mathrm{d} \alpha+\mathrm{d}(\beta(\rho(\alpha)))=\mathrm{d}(\mathrm{d} g(\rho(\mathrm{d} f)))=\mathrm{d}\{f, g\}=\llbracket \mathrm{d} f, \mathrm{~d} g \rrbracket=\llbracket \alpha, \beta \rrbracket .
$$

Since every element of $\Omega^{1}(\mathcal{A})$ is a linear combination of elements of the form $\mathrm{d} f_{i}\left(f_{i} \in \mathcal{A}\right)$, it is enough to prove the statement for $\alpha=f_{1} \mathrm{~d} g_{1}$ and $\beta=f_{2} \mathrm{~d} g_{2}$, which can be done by a direct computation.

The hypothesis of the Proposition 1 can be reformulated in an alternative way.

Proposition 2. If $\left(\Omega^{1}(\mathcal{A}), \llbracket \cdot, \cdot \rrbracket, \rho\right)$ is a Lie algebroid with anchor $\rho$ skew-symmetric, then the following assertions are equivalent:

(i) $\llbracket \mathrm{d} f, \mathrm{~d} g \rrbracket=\mathrm{d}(\mathrm{d} g(\rho(\mathrm{d} f)))=\mathrm{d}\{f, g\}$ for all $f, g \in \mathcal{A}$,

(ii) $\mathrm{d} \alpha=0=\mathrm{d} \beta$ implies $\mathrm{d} \llbracket \alpha, \beta \rrbracket=0$ for $\alpha, \beta \in \Omega^{1}(\mathcal{A})$.

Proof. First, let us assume that item (i) holds. By Proposition 1 we know that the bracket is of the form

$$
\llbracket \alpha, \beta \rrbracket=\iota_{\rho(\alpha)} \mathrm{d} \beta-\iota_{\rho(\beta)} \mathrm{d} \alpha+\mathrm{d}(\beta(\rho(\alpha))),
$$

and hence condition (ii) holds,

$$
\mathrm{d} \llbracket \alpha, \beta \rrbracket=\mathrm{d}\left(\iota_{\rho(\alpha)} \mathrm{d} \beta-\iota_{\rho(\beta)} \mathrm{d} \alpha+\mathrm{d}(\beta(\rho(\alpha)))\right)=\mathrm{d}^{2}(\beta(\rho(\alpha)))=0,
$$

whenever $\mathrm{d} \alpha=0=\mathrm{d} \beta$.

Now let us assume that condition (ii) holds. Define $C(\alpha, \beta)=\llbracket \alpha, \beta \rrbracket-\mathrm{d}(\beta(\rho(\alpha)))$ for $\alpha, \beta \in$ $\Omega^{1}(\mathcal{A})$. Notice that $C$ is skew-symmetric and

$$
\mathrm{d} C(\alpha, \beta)=\mathrm{d}(\llbracket \alpha, \beta \rrbracket)-\mathrm{d}^{2}(\beta(\rho(\alpha)))=0,
$$

for any closed $\alpha$ and $\beta$.

Let us evaluate the following expression

$$
\begin{aligned}
C(\mathrm{~d} f, h \mathrm{~d} h) & =\llbracket \mathrm{d} f, h \mathrm{~d} h \rrbracket-\mathrm{d}(h \mathrm{~d} h(\rho(\mathrm{d} f))) \\
& =h \llbracket \mathrm{d} f, \mathrm{~d} h \rrbracket+\rho(\mathrm{d} f)(\mathrm{d} h) \mathrm{d} h-\mathrm{d} h(\mathrm{~d} h(\rho(\mathrm{d} f)))-h \mathrm{~d}(h \mathrm{~d} h(\rho(\mathrm{d} f))) \\
& =h \llbracket \mathrm{d} f, \mathrm{~d} h \rrbracket-h \mathrm{~d}(h \mathrm{~d} h(\rho(\mathrm{d} f)))=h C(\mathrm{~d} f, \mathrm{~d} h) .
\end{aligned}
$$

Now, applying the operator $\mathrm{d}$ and taking into account that $h \mathrm{~d} h$ is closed, we get

$$
0=\mathrm{d} C(\mathrm{~d} f, h \mathrm{~d} h)=\mathrm{d}(h C(\mathrm{~d} f, \mathrm{~d} h))=\mathrm{d} h \wedge C(\mathrm{~d} f, \mathrm{~d} h)+h \mathrm{~d} C(\mathrm{~d} f, \mathrm{~d} h)=\mathrm{d} h \wedge C(\mathrm{~d} f, \mathrm{~d} h) .
$$

Interchanging the roles of $f$ and $h$, we have

$$
0=\mathrm{d} C(\mathrm{~d} h, f \mathrm{~d} f)=\mathrm{d} f \wedge C(\mathrm{~d} h, \mathrm{~d} f)=-\mathrm{d} f \wedge C(\mathrm{~d} f, \mathrm{~d} h) .
$$

These relations imply that $\mathrm{d} f, \mathrm{~d} h$ and $C(\mathrm{~d} f, \mathrm{~d} h)$ are linearly dependent for arbitrary $f$ and $h$. In particular, if $\mathrm{d} f$ and $\mathrm{d} h$ are linearly independent, then $C(\mathrm{~d} f, \mathrm{~d} h)=0$, and hence

$$
\llbracket \mathrm{d} f, \mathrm{~d} h \rrbracket=\mathrm{d}(\mathrm{d} h(\rho(\mathrm{d} f)))=\mathrm{d}\{f, h\} .
$$

Remark 4. It has been shown that a Poisson bracket $\{\cdot, \cdot\}$ on $M$ defines a graded Poisson bracket $\llbracket \cdot, \cdot \rrbracket$ in $\Omega(M)$ (see [18]). Its values on smooth functions and on exact 1-forms are given by $\llbracket f, g \rrbracket=0, \llbracket \mathrm{d} f, g \rrbracket=\{f, g\}$ and $\llbracket \mathrm{d} f, \mathrm{~d} g \rrbracket=\mathrm{d}\{f, g\}$, respectively. Then, the bracket can be extended to all differential forms by the graded Leibniz rule. For this Poisson bracket the exterior differential is a Poisson derivation, that is,

$$
\mathrm{d} \llbracket \alpha, \beta \rrbracket=\llbracket \mathrm{d} \alpha, \beta \rrbracket+(-1)^{|\alpha|-1} \llbracket \alpha, \mathrm{d} \beta \rrbracket
$$

for any $\alpha, \beta \in \Omega(M)$. Such graded Poisson brackets were called differential in [3]. 
Let us summarize these results.

Theorem 3. Let $\left(\Omega^{1}(\mathcal{A}), \llbracket \cdot, \cdot \rrbracket, \rho\right)$ be a Lie algebroid. Then, there is a Poisson algebra structure $\{\}:, \mathcal{A} \times \mathcal{A} \rightarrow \mathcal{A}$ on $\mathcal{A}$ such that

$$
\rho(\mathrm{d} f)=\operatorname{ad}_{f}, \quad \forall f \in \mathcal{A},
$$

if and only if:

(a) $\rho$ is skew-symmetric

(b) One of the following conditions holds:

1) $\mathrm{d} \alpha=0=\mathrm{d} \beta$ implies $\mathrm{d} \llbracket \alpha, \beta \rrbracket=0$;

2) $\llbracket \mathrm{d} f, \mathrm{~d} g \rrbracket=\mathrm{d}(\mathrm{d} g(\rho(\mathrm{d} f)))$ for all $f, g \in \mathcal{A}$.

Under these conditions, the Lie bracket $\llbracket \cdot, \cdot \rrbracket$ is reconstructed from $\rho$ by the formula

$$
\llbracket \alpha, \beta \rrbracket=\iota_{\rho(\alpha)} \mathrm{d} \beta-\iota_{\rho(\beta)} \mathrm{d} \alpha+\mathrm{d}(\beta(\rho(\alpha))) .
$$

Of course, the basic example of this situation is the cotangent Lie algebroid of a symplectic manifold. A Lie algebroid structure $\left(\Omega^{1}(M), \llbracket \cdot, \cdot \mathbb{\rrbracket}, \rho\right)$ induces a Poisson bracket on $\mathcal{C}^{\infty}(M)$ if and only if $\rho$ is skew-symmetric and, whenever $\mathrm{d} \alpha=0=\mathrm{d} \beta$, then $\mathrm{d} \llbracket \alpha, \beta \rrbracket=0$. For this kind of examples, the property $\llbracket \mathrm{d} f, \mathrm{~d} g \rrbracket=\mathrm{d}\{f, g\}$ follows directly from the injectivity of the anchor map and the fact that it is a Lie algebra morphism: $\rho \llbracket \mathrm{d} f, \mathrm{~d} g \rrbracket=\llbracket \rho(\mathrm{d} f), \rho(\mathrm{d} g) \rrbracket=\rho(\mathrm{d}\{f, g\})$. Let us consider the following example, where the anchor map is not injective but the property $\llbracket \mathrm{d} f, \mathrm{~d} g \rrbracket=\mathrm{d}\{f, g\}$ still holds.

Example 5. Let $\mathcal{R}=\mathbb{R}$ and $\mathcal{A}=\mathbb{R}\left[x^{1}, x^{2}, x^{3}\right]$. Then, $\operatorname{Der}(\mathcal{A})=\operatorname{Span}\left\{\partial_{1}, \partial_{2}, \partial_{2}\right\}$ and $\Omega^{1}(\mathcal{A})=$ $\operatorname{Span}\left\{\mathrm{d} x^{1}, \mathrm{~d} x^{2}, \mathrm{~d} x^{3}\right\}$. Define the following bracket

$$
\begin{aligned}
\llbracket p_{i} \mathrm{~d} x^{i}, q_{j} \mathrm{~d} x^{j} \rrbracket= & \left(\left(p_{1}\left(\partial_{2}+\partial_{3}\right)+p_{2}\left(-\partial_{1}+\partial_{3}\right)+p_{3}\left(-\partial_{1}-\partial_{2}\right)\right)\left(q_{i}\right)\right. \\
& \left.-\left(q_{1}\left(\partial_{2}+\partial_{3}\right)+q_{2}\left(-\partial_{1}+\partial_{3}\right)+q_{3}\left(-\partial_{1}-\partial_{2}\right)\right)\left(p_{i}\right)\right) \mathrm{d} x^{i},
\end{aligned}
$$

and the anchor map as

$$
\begin{aligned}
\rho: & \Omega^{1}(\mathcal{A}) \rightarrow \operatorname{Der}(\mathcal{A}) \\
& p_{i} \mathrm{~d} x^{i} \mapsto-\left(p_{2}+p_{3}\right) \partial_{1}+\left(p_{1}-p_{3}\right) \partial_{2}+\left(p_{1}+p_{2}\right) \partial_{3} .
\end{aligned}
$$

Note that the matrix representation of $\rho$ relative to the given basis in $\operatorname{Der}(\mathcal{A})$ and $\Omega^{1}(\mathcal{A})$ is

$$
\rho=\left(\begin{array}{ccc}
0 & 1 & 1 \\
-1 & 0 & 1 \\
-1 & -1 & 0
\end{array}\right)
$$

Therefore, $\rho$ is skew-symmetric and of rank 2 ( $\rho$ is not injective). A long but straightforward computation shows that $\left(\Omega^{1}(\mathcal{A}),\right][\cdot, \cdot \rrbracket, \rho)$ is a Lie algebroid.

Let us show that this Lie algebroid is of Poisson type by checking the property $\llbracket d f, d g \rrbracket=$ $d\{f, g\}$. We have for $p, q \in \mathcal{A}$ :

$$
\begin{aligned}
\{p, q\} & =\mathrm{d}(\rho(\mathrm{d} p)(q))=\mathrm{d}\left(\rho\left(\partial_{i} p \mathrm{~d} x^{i}\right)(q)\right) \\
& =\mathrm{d}\left(-\left(\partial_{2} p+\partial_{3} p\right) \partial_{1} q+\left(\partial_{1} p-\partial_{3} p\right) \partial_{2} q+\left(\partial_{1} p+\partial_{2} p\right) \partial_{3} q\right) .
\end{aligned}
$$


The $\mathrm{d} x^{1}$ factor in the expansion of this expression (the other cases are similar) is

$$
\begin{aligned}
& -\left(\partial_{2} p+\partial_{3} p\right) \partial_{11}^{2} q+\left(\partial_{1} p-\partial_{3} p\right) \partial_{12}^{2} q+\left(\partial_{1} p+\partial_{2} p\right) \partial_{13}^{2} q \\
& \quad-\left(\partial_{12}^{2} p+\partial_{13}^{2} p\right) \partial_{1} q+\left(\partial_{11}^{2} p-\partial_{13}^{2} p\right) \partial_{2} q+\left(\partial_{11}^{2} p+\partial_{12}^{2} p\right) \partial_{3} q .
\end{aligned}
$$

On the other hand, the Lie algebroid bracket is

$$
\left.\llbracket \mathrm{d} p, \mathrm{~d} q \rrbracket=\llbracket \partial_{i} p \mathrm{~d} x^{i}, \partial_{j} q \mathrm{~d} x^{j} \rrbracket=\rho(\mathrm{d} p)\left(\partial_{k} q\right)-\rho(\mathrm{d} q)\left(\partial_{k} p\right)\right) \mathrm{d} x^{k} .
$$

For $k=1$, we compute the coefficient of $\mathrm{d} x^{1}$ :

$$
\begin{aligned}
& -\left(\partial_{2} p+\partial_{3} p\right) \partial_{11}^{2} q+\left(\partial_{1} p-\partial_{3} p\right) \partial_{12}^{2} q+\left(\partial_{1} p+\partial_{2} p\right) \partial_{13}^{2} q \\
& \quad+\left(\partial_{2} q+\partial_{3} q\right) \partial_{11}^{2} p-\left(\partial_{1} q-\partial_{3} q\right) \partial_{12}^{2} p-\left(\partial_{1} q+\partial_{2} q\right) \partial_{13}^{2} p,
\end{aligned}
$$

which is the same as above. Thus, $\left(\Omega^{1}(\mathcal{A}), \llbracket \cdot, \cdot \rrbracket, \rho\right)$ is a Lie algebroid of Poisson type and its bracket is just given by formula (3).

Remark 5. The anchor map of the cotangent Lie algebroid is not injective in general. It is only true in the symplectic case.

Finally, let us consider an example of Lie algebroid of Poisson type whose bracket does not have the form (3).

Example 6. Let $\mathcal{R}=\mathbb{R}$ and $\mathcal{A}=\mathbb{R}\left[x^{1}, x^{2}, x^{3}\right]$. Define the structure of an Abelian Lie algebra with generators $\left\{\mathrm{d} x^{1}, \mathrm{~d} x^{2}, \mathrm{~d} x^{3}\right\}$,

$$
\llbracket \mathrm{d} x^{i}, \mathrm{~d} x^{j} \rrbracket=0, \quad i, j \in\{1,2,3\} .
$$

Extending by $\mathcal{R}$-bilinearity and the Leibniz identity gives

$$
\llbracket p_{i} \mathrm{~d} x^{i}, q_{j} \mathrm{~d} x^{j} \rrbracket=p_{i} \rho\left(\mathrm{d} x^{i}\right)\left(q_{j}\right) \mathrm{d} x^{j}-q_{j} \rho\left(\mathrm{d} x^{j}\right)\left(p_{i}\right) \mathrm{d} x^{i} .
$$

Next, let us think of the following skew-symmetric morphism of $\mathcal{A}$-modules as the anchor map:

$$
\begin{aligned}
\rho: & \Omega^{1}(\mathcal{A}) \rightarrow \operatorname{Der}(\mathcal{A}), \\
& \mathrm{d} x^{1} \mapsto x^{3} \partial_{2}, \\
\mathrm{~d} x^{2} & \mapsto-x^{3} \partial_{1}, \\
\mathrm{~d} x^{3} & \mapsto 0 .
\end{aligned}
$$

With these definitions the bracket in $\Omega^{1}(\mathcal{A})$ can also be expressed in a form suitable for explicit computations, as

$$
\llbracket p_{i} \mathrm{~d} x^{i}, p_{j} \mathrm{~d} x^{j} \rrbracket=x^{3}\left((p \times \nabla)_{3} q_{i}-(q \times \nabla)_{3} p_{i}\right) \mathrm{d} x^{i},
$$

where an element $p \in \mathcal{A}$ is viewed as a vector $p=\left(p_{1}, p_{2}, p_{3}\right), \nabla=\left(\partial_{1}, \partial_{2}, \partial_{3}\right)$ and the subindex 3 denotes the third component of the cross product $p \times \nabla$.

It can be checked that $\left(\Omega^{1}(\mathcal{A}), \llbracket \cdot, \cdot \rrbracket, \rho\right)$ is a Lie algebroid of Poisson type. However, if $p=$ $2 x^{1}+x^{2}$ and $q=x^{1}+x^{2}$, then

$$
\llbracket \mathrm{d} p, \mathrm{~d} q \rrbracket=\llbracket 2 \mathrm{~d} x^{1}+\mathrm{d} x^{2}, \mathrm{~d} x^{1}+\mathrm{d} x^{2} \rrbracket=0,
$$

whereas

$$
\begin{aligned}
\mathrm{d}\{p, q\}=\mathrm{d}(\mathrm{d} q(\rho(\mathrm{d} p))) & =\mathrm{d}\left(\left(\mathrm{d} x^{1}+\mathrm{d} x^{2}\right) \rho\left(2 \mathrm{~d} x^{1}+\mathrm{d} x^{2}\right)\right) \\
& =\mathrm{d}\left(\left(\mathrm{d} x^{1}+\mathrm{d} x^{2}\right)\left(2 x^{3} \partial_{2}-x^{3} \partial_{1}\right)\right)=\mathrm{d} x^{3} .
\end{aligned}
$$




\section{Transitive Lie algebroids}

To motivate the definition of transitive Lie algebroids let us consider for a moment the geometric example of a Lie algebroid $(E, \llbracket \cdot, \cdot \rrbracket, q)$, where $E \rightarrow M$ is a vector bundle over a manifold $M$ (recall Example 1). If the anchor map $q: \Gamma(E) \rightarrow \Gamma(T M)$ is an epimorphism, the algebroid $(E, \llbracket \cdot, \rrbracket, q)$ is said to be transitive. In this case, it is possible to construct the so-called Atiyah sequence of the algebroid, which is the short exact sequence

$$
\mathfrak{g} \stackrel{j}{\longrightarrow} E \stackrel{q}{\longrightarrow} T M
$$

where $\mathfrak{g}=\operatorname{Ker} q$. Thus, the existence of a section for the anchor $q$ (equivalently, a linear connection on $E$ ) implies that, locally, $E=T M \oplus \mathfrak{g}$. Note also that the fibre of the bundle $\mathfrak{g}$ over the point $x \in M, \mathfrak{g}_{x}$ is a Lie algebra (called the isotropy Lie algebra of the algebroid $E$ at $x \in M)$ with the bracket given, for $\alpha, \beta \in \mathfrak{g}_{x}$, by

$$
[\alpha, \beta]=\llbracket X, Y \rrbracket,
$$

where $X, Y \in \Gamma(E)$ are any sections such that $X(x)=\alpha$ and $Y(x)=\beta$.

Definition 9. A Lie algebroid $(\mathcal{F}, \llbracket \cdot, \cdot \rrbracket, \rho)$ is transitive if there exist a short exact sequence of $\mathcal{A}$-modules:

$$
V \stackrel{j}{\longrightarrow} \mathcal{F} \stackrel{\rho}{\longrightarrow} \operatorname{Der}(\mathcal{A})
$$

Remark 6. There is a more general notion, the extension of a Lie-Rinehart algebra, that generalizes the transitivity condition for a geometric Lie algebroid (see [13]).

\subsection{Transitive algebroids induced by connections}

Let $V$ be a unitary $\mathcal{A}$-module and $\nabla$ a connection on $V$.

Definition 10. The curvature of $\nabla$ is the mapping $C_{\nabla}: \operatorname{Der}(\mathcal{A}) \times \operatorname{Der}(\mathcal{A}) \rightarrow \operatorname{Hom}(V, V)$ defined by

$$
C_{\nabla}(X, Y)=\left[\nabla_{X}, \nabla_{Y}\right]-\nabla_{[X, Y]}
$$

Definition 11. If $V$ is endowed with a Lie algebra structure $[\cdot, \cdot]_{V}$, then a connection $\nabla$ is said to be a Lie connection if

$$
\nabla_{X}\left[v_{1}, v_{2}\right]_{V}=\left[\nabla_{X} v_{1}, v_{2}\right]_{V}+\left[v_{1}, \nabla_{X} v_{2}\right]_{V}
$$

for all $v_{1}, v_{2} \in V$ and for all $X \in \operatorname{Der}(\mathcal{A})$.

We have the following result, which gives us a description of transitive Lie algebroids in terms of Lie connections (see, also [20, 21]).

Theorem 4. Let $V$ be an $\mathcal{A}$-module endowed with a Lie algebra structure $[,]_{V}$ which is $\mathcal{A}$ linear (i.e., $\left.\left[f v_{1}, v_{2}\right]_{V}=f\left[v_{1}, v_{2}\right]_{V} \forall f \in \mathcal{A}, v_{1}, v_{2} \in V\right)$. Let $\nabla$ be a Lie connection on $V$. If there exists a 2 -form $\mathcal{B} \in \Omega^{2}(\mathcal{A} ; V)$ with values in $V$, such that, for any $X_{1}, X_{2}, X_{3} \in \operatorname{Der}(\mathcal{A})$ and $v \in V$ the following conditions hold:

(a) $\left[\mathcal{B}\left(X_{1}, X_{2}\right), v\right]_{V}=C_{\nabla}\left(X_{1}, X_{2}\right)(v)$,

(b) the cyclic sum $\circlearrowleft\left(\nabla_{X_{1}}\left(\mathcal{B}\left(X_{2}, X_{3}\right)\right)-\mathcal{B}\left(\left[X_{1}, X_{2}\right], X_{3}\right)\right)=0$, 
then $(\operatorname{Der}(\mathcal{A}) \oplus V, \llbracket \cdot, \cdot \rrbracket, \rho)$ is a transitive Lie algebroid with anchor map $\rho=\operatorname{pr}_{1}$ and bracket

$$
\llbracket\left(X_{1}, v_{1}\right),\left(X_{2}, v_{2}\right) \rrbracket=\left(\left[X_{1}, X_{2}\right],\left[v_{1}, v_{2}\right]_{V}+\nabla_{X_{1}} v_{2}-\nabla_{X_{2}} v_{1}-\mathcal{B}\left(X_{1}, X_{2}\right)\right) .
$$

Moreover,

$$
\iota_{2}(\mathcal{B}(X, Y))=\iota_{1}([X, Y])-\llbracket \iota_{1}(X), \iota_{1}(Y) \rrbracket,
$$

for $X, Y \in \operatorname{Der}(\mathcal{A})$. Here $\iota_{1}: \operatorname{Der}(\mathcal{A}) \rightarrow \operatorname{Der}(\mathcal{A}) \oplus V$ and $\iota_{2}: V \rightarrow \operatorname{Der}(\mathcal{A}) \oplus V$ are the inclusion maps.

Proof. It is clear that $\operatorname{Der}(\mathcal{A}) \oplus V$ is an $\mathcal{A}$-module. We must show that the bracket defined by (5) is Lie. The skew-symmetry and the $\mathcal{R}$-bilinearity are immediate, while the Jacobi identity and the Leibniz rule are checked by lengthy but straightforward computations. Since $\rho=\operatorname{pr}_{1}$ is clearly $\mathcal{A}$-linear, we have that $\operatorname{Der}(\mathcal{A}) \oplus V$ is a Lie algebroid. The transitivity is obvious in view of the sequence

$$
V \stackrel{\iota_{2}}{\longrightarrow} \operatorname{Der}(\mathcal{A}) \oplus V \stackrel{\iota_{1}}{\longrightarrow} \operatorname{Der}(\mathcal{A}) .
$$

Moreover, a direct computation shows that

$$
\begin{aligned}
\iota_{1}[X, Y]-\llbracket \iota_{1}(X), \iota_{1}(Y) \rrbracket & =([X, Y], 0)-\llbracket(X, 0),(Y, 0) \rrbracket=([X, Y], 0)-([X, Y],-\mathcal{B}(X, Y)) \\
& =(0, \mathcal{B}(X, Y))=\iota_{2}(\mathcal{B}(X, Y)) .
\end{aligned}
$$

Remark 7. The conditions (a) and (b) in this theorem have the following interpretation. Condition (a) states that the curvature of the connection $\nabla$ is given by the composition $C_{\nabla}=\operatorname{ad}^{R} \circ \mathcal{B}$, where $\operatorname{ad}^{R}: V \rightarrow V$ is the right adjoint with respect to the bracket on $V$. On the other hand, (b) expresses the Bianchi identity for a connection with torsion (see also [20, 21]).

\subsection{Parametrization of transitive algebroids on $\operatorname{Der} \mathcal{A} \oplus V$}

The following result states that the converse of Theorem 4 is also true.

Theorem 5. Let $(\operatorname{Der}(\mathcal{A}) \oplus V, \llbracket \cdot, \cdot \rrbracket, \rho)$ be a transitive Lie algebroid with $\rho=\operatorname{pr}_{1}$. Then:

(i) The bracket on $V$, defined for $v_{1}, v_{2} \in V$ by

$$
\left[v_{1}, v_{2}\right]_{V}=\operatorname{pr}_{2}\left(\llbracket \iota_{2}\left(v_{1}\right), \iota_{2}\left(v_{2}\right) \rrbracket\right),
$$

is an $\mathcal{A}$-linear Lie bracket.

(ii) The mapping $\nabla: \operatorname{Der}(\mathcal{A}) \rightarrow \operatorname{Hom}(V, V)$ given by

$$
\nabla_{X}(v)=\operatorname{pr}_{2}\left(\llbracket \iota_{1}(X), \iota_{2}(v) \rrbracket\right),
$$

for $v \in V$ and $X \in \operatorname{Der}(\mathcal{A})$, is a Lie connection on $V$.

(iii) The mapping $\mathcal{B}: \operatorname{Der}(\mathcal{A}) \times \operatorname{Der}(\mathcal{A}) \rightarrow V$ defined by

$$
\mathcal{B}(X, Y)=\operatorname{pr}_{2}\left(\llbracket \iota_{1}(X), \iota_{1}(Y) \rrbracket-\iota_{1}([X, Y])\right),
$$

is $\mathcal{A}$-bilinear, skew-symmetric and it satisfies conditions (a) and (b) of Theorem 4.

Proof. The proof is a lengthy but straightforward computation developing the definitions.

As a consequence of Theorems 4 and 5, we have the following.

Corollary 1. Let $V$ be an $\mathcal{A}$-module. There is a one to one correspondence between transitive Lie algebroids $(\operatorname{Der}(\mathcal{A}) \oplus V, \llbracket, \rrbracket, \rho)$ with anchor $\rho=\operatorname{pr}_{1}$ and triples $\left([\cdot, \cdot]_{V}, \nabla, \mathcal{B}\right)$ consisting of:

(i) an $\mathcal{A}$-linear Lie bracket $[\cdot, \cdot]_{V}$ in $V$;

(ii) a Lie connection $\nabla$ on $V$;

(iii) a 2-form $\mathcal{B} \in \Omega^{2}(\mathcal{A} ; V)$ satisfying conditions (a) and (b) of Theorem 4. 


\subsection{Algebroid connections}

In this subsection, we first generalize Theorem 5 to the case of a transitive Lie algebroid which is not necessarily of the form $\operatorname{Der}(\mathcal{A}) \oplus V$, but we require that it be endowed with a Lie algebroid connection (see [19, 21]), considered as a section of the anchor map. Then, in Theorem 6 we construct a Lie algebroid structure on $\operatorname{Der}(\mathcal{A}) \oplus V$ which is isomorphic to the given algebroid.

Definition 12. Let $(\mathcal{F}, \llbracket \cdot, \cdot \rrbracket, \rho)$ be a Lie algebroid. A morphism of $\mathcal{A}$-modules $\gamma: \operatorname{Der}(\mathcal{A}) \rightarrow \mathcal{F}$ is called a Lie algebroid connection $\left(\right.$ on $\mathcal{F}$ ) if it is a section of $\rho$, that is, $\rho \circ \gamma=\operatorname{Id}_{\operatorname{Der}(\mathcal{A})}$.

As stated in Section 2.1, we will assume some condition guaranteeing the existence of a connection on $(\mathcal{F}, \llbracket \cdot, \cdot \rrbracket, \rho)$, to be precise, we will assume that $\mathcal{F}$ is free. It is known that the existence of a section $\gamma$ of $\rho$ in the short exact sequence

$$
V \stackrel{\iota}{\longrightarrow} \mathcal{F} \stackrel{\rho}{\longrightarrow} \operatorname{Der}(\mathcal{A})
$$

implies that $\mathcal{F} \cong V \oplus \operatorname{Der}(\mathcal{A})$.

Proposition 3. Let

$$
V \stackrel{\iota}{\longrightarrow} \mathcal{F} \stackrel{\rho}{\longrightarrow} \operatorname{Der}(\mathcal{A})
$$

be a transitive Lie algebroid and $\gamma: \operatorname{Der}(\mathcal{A}) \rightarrow \mathcal{F}$ a Lie algebroid connection for $\mathcal{F}$. Then:

(i) The bracket $[\cdot, \cdot]_{V}$ defined for $v_{1}, v_{2} \in V$ by

$$
\left[v_{1}, v_{2}\right]_{V}=\llbracket \iota\left(v_{1}\right), \iota\left(v_{2}\right) \rrbracket,
$$

is an $\mathcal{A}$-linear Lie bracket.

(ii) The mapping $\nabla: \operatorname{Der}(\mathcal{A}) \rightarrow \operatorname{Hom}(V, V)$ given by

$$
\nabla_{X}(v)=\llbracket \gamma(X), \iota(v) \rrbracket,
$$

is a Lie connection on $V$ (called the adjoint connection).

(iii) The mapping $\mathcal{B}: \operatorname{Der}(\mathcal{A}) \times \operatorname{Der}(\mathcal{A}) \rightarrow V$, defined by

$$
\mathcal{B}(X, Y)=\llbracket \gamma(X), \gamma(Y) \rrbracket-\gamma([X, Y]),
$$

is $\mathcal{A}$-bilinear, skew-symmetric and satisfies the conditions (a) and (b) of Theorem 4.

Proof. First, note that the exactness of (6) allows us to identify $V$ with its image under $\iota$ in $\mathcal{F}$, and that $\operatorname{Im} \iota=\operatorname{ker} \rho$ in $\mathcal{F}$. Then, since $\rho$ is a morphism of Lie algebras, we have

$$
\rho \llbracket \iota\left(v_{1}\right), \iota\left(v_{2}\right) \rrbracket=\left[\rho \iota\left(v_{1}\right), \rho \iota\left(v_{2}\right)\right]=0 .
$$

Thus, the bracket $[\cdot, \cdot]_{V}$ is well-defined. By a similar argument it can be proved that $\nabla$ and $\mathcal{B}$

are well-defined. The proof of the remaining properties follows the same guidelines that those in Theorem 5.

Theorem 6. Let

$$
V \stackrel{\iota}{\longrightarrow} \mathcal{F} \stackrel{\rho}{\longrightarrow} \operatorname{Der}(\mathcal{A})
$$

be a transitive Lie algebroid and $\gamma: \operatorname{Der}(\mathcal{A}) \rightarrow \mathcal{F}$ a Lie algebroid connection on $\mathcal{F}$. Then $(\mathcal{F}, \llbracket \cdot, \cdot \rrbracket, \rho)$ is isomorphic to $\left(\operatorname{Der}(\mathcal{A}) \oplus V,\langle\cdot, \cdot\rangle, \operatorname{pr}_{1}\right)$, where the bracket is defined through

$$
\left\langle\left(X_{1}, v_{1}\right),\left(X_{2}, v_{2}\right)\right\rangle=\left(\left[X_{1}, X_{2}\right],\left[v_{1}, v_{2}\right]_{V}+\nabla_{X_{1}}\left(v_{2}\right)-\nabla_{X_{2}}\left(v_{1}\right)-\mathcal{B}\left(X_{1}, X_{2}\right)\right),
$$

and $[\cdot, \cdot]_{V}, \nabla_{X}, \mathcal{B}$ are given in Proposition 3. 
Proof. We know that $\left(\operatorname{Der}(\mathcal{A}) \oplus V,\langle\cdot, \cdot\rangle, \operatorname{pr}_{1}\right)$ is a Lie algebroid by Theorem 4 . Moreover,

$$
\begin{aligned}
\phi: & \operatorname{Der}(\mathcal{A}) \oplus V \longrightarrow \mathcal{F}, \\
& (X, v) \longmapsto \gamma(X)+\iota(v)
\end{aligned}
$$

is an $\mathcal{A}$-module isomorphism such that

$$
(\rho \circ \phi)(X, v)=\rho(\gamma(X)+\iota(v))=X=\operatorname{pr}_{1}(X, v)
$$

and, by a straightforward computation,

$$
\phi\left(\left\langle\left(X_{1}, v_{1}\right),\left(X_{2}, v_{2}\right)\right\rangle\right)=\llbracket \phi\left(X_{1}, v_{1}\right), \phi\left(X_{2}, v_{2}\right) \rrbracket .
$$

\section{Poisson algebras on $\mathcal{A} \oplus V$}

Recall that, if $(\mathcal{A},\{\}$,$) is a Poisson algebra, then we define for every f \in \mathcal{A}$ the Hamiltonian derivation $X_{f} \in \operatorname{Der}(\mathcal{A})$ as the adjoint map $X_{f}=\{f, \cdot\}$. The following standard properties will be used below:

(i) $X_{f_{1} f_{2}}=f_{1} X_{f_{2}}+f_{2} X_{f_{1}}$,

(ii) the mapping $f \mapsto X_{f}$ is a Lie algebra morphism, i.e., $X_{\left\{f_{1}, f_{2}\right\}}=\left[X_{f_{1}}, X_{f_{2}}\right]$.

Now, on $\mathcal{A} \oplus V$ we can define a product given by

$$
\left(f_{1}, v_{1}\right) \cdot\left(f_{2}, v_{2}\right)=\left(f_{1} f_{2}, f_{1} v_{2}+f_{2} v_{1}\right),
$$

for $f_{1}, f_{2} \in \mathcal{A}$ and $v_{1}, v_{2} \in V$. This makes $\mathcal{A} \oplus V$ a commutative ring. Under certain conditions, this ring is also a Poisson algebra, as shown by the following result.

Theorem 7. Let $\left(\mathcal{A},\{,\}_{\mathcal{A}}\right)$ be a Poisson algebra. Suppose we have a transitive Lie algebroid

$$
V \stackrel{\iota_{2}}{\longrightarrow} \operatorname{Der}(\mathcal{A}) \oplus V \stackrel{\rho}{\longrightarrow} \operatorname{Der}(\mathcal{A}),
$$

with anchor $\rho=\operatorname{pr}_{1}$. Then, $\mathcal{A} \oplus V$ is also a Poisson algebra with product defined by (7) and Poisson bracket

$$
\left\{\left(f_{1}, v_{1}\right),\left(f_{2}, v_{2}\right)\right\}=\left(\left\{f_{1}, f_{2}\right\}_{\mathcal{A}}, \nabla_{X_{f_{1}}}\left(v_{2}\right)-\nabla_{X_{f_{2}}}\left(v_{1}\right)+\left[v_{1}, v_{2}\right]_{V}+\mathcal{B}\left(X_{f_{1}}, X_{f_{2}}\right)\right),
$$

where $[\cdot, \cdot]_{V}, \nabla$ and $\mathcal{B}$ are given in Theorem 5 .

Proof. The Jacobi identity and the Leibniz rule are proved by direct computations, making use of the $\mathcal{A}$-linearity of the bracket $[,]_{V}$.

Corollary 2. $V$ is an ideal of $\mathcal{A} \oplus V$ with respect to the Poisson bracket defined by (8).

The proof of this result follows from the fact $\{0, f\}_{\mathcal{A}}=0$.

Corollary 3. If $\left(\mathcal{A},\{,\}_{\mathcal{A}}\right)$ is a Poisson algebra and we have a transitive Lie algebroid

$$
V \stackrel{\iota_{2}}{\longrightarrow} \mathcal{F} \stackrel{\rho}{\longrightarrow} \operatorname{Der}(\mathcal{A})
$$

endowed with an algebroid connection $\gamma$, then, $\mathcal{A} \oplus V$ is a Poisson algebra.

Proof. By Theorem 6, we know that $(\mathcal{F}, \llbracket \cdot, \cdot \rrbracket, \rho)$ is isomorphic to $\left(\operatorname{Der}(\mathcal{A}) \oplus V,\langle\cdot, \cdot\rangle, \operatorname{pr}_{1}\right)$. The statement then follows from Theorem 7 . 


\section{Acknowledgements}

JAV and DGB express their gratitude to the members of the Department of Mathematics of the University of Sonora (where part of this work was done), particularly to R. Flores-Espinoza and G. Dávila-Rascón, for their warm hospitality. JAV also thanks the National Council of Science and Technology in Mexico (CONACyT), for the research grant JB2-CB-78791.

\section{References}

[1] Abraham R., Marsden J.E., Kelley A., Kolmogorov A.N., Foundations of mechanics, W.A. Benjamin, Inc., New York - Amsterdam, 1967.

[2] Atkin C.J., Grabowski J., Homomorphisms of the Lie algebras associated with a symplectic manifold, Compositio Math. 76 (1990), 315-349.

[3] Beltrán J.V., Monterde J., Graded Poisson structures on the algebra of differential forms, Comment. Math. Helv. 70 (1995), 383-402.

[4] Connes A., Noncommutative differential geometry, Inst. Hautes Études Sci. Publ. Math. (1985), $257-360$.

[5] Cuntz J., Quillen D., Algebra extensions and nonsingularity, J. Amer. Math. Soc. 8 (1995), 251-289.

[6] Dubois-Violette M., Michor P.W., Dérivations et calcul différentiel non commutatif. II, C. R. Acad. Sci. Paris Sér. I Math. 319 (1994), 927-931.

[7] Dufour J.P., Zung N.T., Poisson structures and their normal forms, Progress in Mathematics, Vol. 242, Birkhäuser Verlag, Basel, 2005.

[8] Grabowski J., Quasi-derivations and QD-algebroids, Rep. Math. Phys. 52 (2003), 445-451, math.DG/0301234.

[9] Herz J.C., Pseudo-algèbres de Lie. I, C. R. Acad. Sci. Paris 236 (1953), 1935-1937.

[10] Herz J.C., Pseudo-algèbres de Lie. II, C. R. Acad. Sci. Paris 236 (1953), 2289-2291.

[11] Huebschmann J., Poisson cohomology and quantization, J. Reine Angew. Math. 408 (1990), 57-113.

[12] Huebschmann J., Lie-Rinehart algebras, Gerstenhaber algebras and Batalin-Vilkovisky algebras, Ann. Inst. Fourier (Grenoble) 48 (1998), 425-440, dg-ga/9704005.

[13] Huebschmann J., Extensions of Lie-Rinehart algebras and the Chern-Weil construction, in Higher Homotopy Structures in Topology and Mathematical Physics (Poughkeepsie, NY, 1996), Contemp. Math., Vol. 227, Amer. Math. Soc., Providence, RI, 1999, 145-176, dg-ga/9706002.

[14] Katz N.M., Nilpotent connections and the monodromy theorem: applications of a result of Turrittin, Inst. Hautes Études Sci. Publ. Math. (1970), no. 39, 175-232.

[15] Kolář I., Michor P.W., Slovák J., Natural operations in differential geometry, Springer-Verlag, Berlin, 1993.

[16] Kosmann-Schwarzbach Y., Mackenzie K.C.H., Differential operators and actions of Lie algebroids, in Quantization, Poisson Brackets and Beyond (Manchester, 2001), Contemp. Math., Vol. 315, Amer. Math. Soc., Providence, RI, 2002, 213-233, math.DG/0209337.

[17] Kosmann-Schwarzbach Y., Magri F., Poisson-Nijenhuis structures, Ann. Inst. H. Poincaré Phys. Théor. 53 (1990), 35-81.

[18] Koszul J.L., Crochet de Schouten-Nijenhuis et cohomologie, Astérisque (1985), Numero Hors Serie, $257-271$.

[19] Kubarski J., The Chern-Weil homomorphism of regular Lie algebroids, Publications du Département de Mathématiques, Nouvelle Série A, Univ. Claude-Bernard, Lyon, 1991, 1-70.

[20] Mackenzie K.C.H., Lie algebroids and Lie pseudoalgebras, Bull. London Math. Soc. 27 (1995), 97-147.

[21] Mackenzie K.C.H., General theory of Lie groupoids and Lie algebroids, London Mathematical Society Lecture Note Series, Vol. 213, Cambridge University Press, Cambridge, 2005.

[22] Rinehart G.S., Differential forms on general commutative algebras, Trans. Amer. Math. Soc. 108 (1963), $195-222$.

[23] Vorobiev Yu., On Poisson realizations of transitive Lie algebroids, J. Nonlinear Math. Phys. 11 (2004), suppl., 43-48.

[24] Vorobjev Yu., Coupling tensors and Poisson geometry near a single symplectic leaf, in Lie Algebroids and Related Topics in Differential Geometry (Warsaw, 2000), Banach Center Publ., Vol. 54, Polish Acad. Sci. Inst. Math., Warsaw, 2001, 249-274, math.SP/0008162. 